\title{
Etravirine (TMC-125): The evidence for its place in the treatment of HIV-I infection
}

This article was published in the following Dove Press journal:

Core Evidence

2 I July 2009

Number of times this article has been viewed

\section{Hans-Jürgen Stellbrink}

Infektionsmedizinisches Centrum Hamburg (ICH), Hamburg, Germany
Correspondence: Hans-Jürgen Stellbrink Infektionsmedizisches Centrum Hamburg (ICH), Grindelallee 35, 20146 Hamburg, Germany Email stellbrink@ich-hamburg.de
Introduction: Etravirine is a novel nonnucleoside reverse transcriptase inhibitor (NNRTI) specifically designed to suppress the replication of viruses resistant to the three currently approved NNRTIs efavirenz, nevirapine, and delavirdine.

Aims: To assess the evidence for the place of etravirine in the treatment of HIV-1 infection.

Evidence review: In combination with a ritonavir-boosted protease inhibitor etravirine has demonstrated high antiviral activity against strains exhibiting up to three NNRTI resistance mutations. The drug appears to be well tolerated, with only nausea and rash occuring significantly more frequently with etravirine compared with placebo. Of note, neuropsychologic side effects that frequently limit the use of efavirenz were not reported more frequently with etravirine.

Place in therapy: Given its high activity against most NNRTI-resistant strains and its very good tolerability, etravirine is of high value for pretreated patients with NNRTI resistance and protease inhibitor exposure. Efforts should be made to demonstrate activity in switching strategies (due to toxicity) and earlier lines of failure or in the setting of primary NNRTI resistance in order to explore the potential of the drug beyond salvage therapy.

Keywords: etravirine, HIV-1, evidence

Core evidence place in therapy summary for etravirine treatment in HIV-I infection

\begin{tabular}{|c|c|c|}
\hline Outcome measure & Evidence & Implications \\
\hline \multicolumn{3}{|l|}{ Patient-oriented evidence } \\
\hline Improvement of survival & Some & $\begin{array}{l}\text { Superior viral load reduction and CD4+ } \\
\text { T-cell increase most likely results in } \\
\text { survival benefit (generally accepted } \\
\text { surrogate markers) }\end{array}$ \\
\hline $\begin{array}{l}\text { Absence of cutaneous allergic } \\
\text { reactions }\end{array}$ & Limited & $\begin{array}{l}\text { Rash also observed with etravirine, no } \\
\text { direct comparison with other NNRTI }\end{array}$ \\
\hline $\begin{array}{l}\text { Absence of neuropsychiatric } \\
\text { side effects }\end{array}$ & Clear & $\begin{array}{l}\text { Frequency of neuropsychiatric side } \\
\text { effects with etravirine same as with } \\
\text { placebo }\end{array}$ \\
\hline $\begin{array}{l}\text { Few treatment } \\
\text { discontinuations }\end{array}$ & Clear & $\begin{array}{l}\text { Side effects infrequent and virologic } \\
\text { failure less likely with etravirine }\end{array}$ \\
\hline \multicolumn{3}{|l|}{ Disease-oriented evidence } \\
\hline Superior viral load suppression & Clear & $\begin{array}{l}\text { Etravirine use associated with improved } \\
\text { virus suppression }\end{array}$ \\
\hline $\begin{array}{l}\text { Superior CD4+ T-cell } \\
\text { reconstitution }\end{array}$ & Clear & $\begin{array}{l}\text { Etravirine use associated with superior } \\
\text { increase in CD4+T-cell counts }\end{array}$ \\
\hline & & (Continued) \\
\hline
\end{tabular}




\begin{tabular}{|c|c|c|}
\hline \multicolumn{3}{|l|}{ (Continued) } \\
\hline Outcome measure & Evidence & Implications \\
\hline \multicolumn{3}{|l|}{ Economic evidence } \\
\hline $\begin{array}{l}\text { Cost-effectiveness in salvage } \\
\text { therapy }\end{array}$ & Limited & $\begin{array}{l}\text { Benefit probable, but not enough } \\
\text { evidence }\end{array}$ \\
\hline $\begin{array}{l}\text { Cost-effectiveness in early } \\
\text { therapy }\end{array}$ & No evidence & $\begin{array}{l}\text { More costly than efavirenz and } \\
\text { nevirapine, more evidence required }\end{array}$ \\
\hline
\end{tabular}

\section{Scope, aims, and objectives}

Etravirine was licensed by the FDA in January 2008 under the trade name Intelence ${ }^{\mathrm{TM}} .^{1}$ It is currently licensed in the US, Canada, and Switzerland, and in the European Union. It belongs to the chemical class of diarylpyrimidine compounds and acts as a nonnucleoside reverse transcriptase inhibitor (NNRTI), which inhibits the enzyme in a noncompetitive way. The aim of this article is to summarize current knowledge about the development of etravirine and to discuss its role in the treatment of HIV-1 infection.

\section{Methods}

The medical literature and the meeting abstracts of major scientific conferences on infectious diseases since January 1, 2003 were reviewed with regard to contributions on etravirine (formerly TMC125 or R165335, manufactured by Tibotec, a division of Janssen Cilag). In view of the rapid advances in the field of HIV treatment, meeting abstracts and internet communications were also included. PubMed (http://www.ncbi.nlm.nih.gov/sites/entrez) was searched using the search term "etravirine or TMC125 or TMC125." In addition, homepages of major conferences on HIV infection and infectious diseases (www. retroconference. org; www.iasociety.org/AbstractSearch.aspx; HIV Drug Resistance Workshop 2008: http://www.hivpresentation. com/index.cfm; European AIDS Clinical Society: http:// www.eacs.eu/index_ns.htm; Interscience Conference on Antimicrobial Agents and Chemotherapy: http://www.icaac. org/) were searched for abstracts including the search term. If primary scientific sources were unavailable, information compiled in company documents was added. Table 1 shows the results of the literature search and the records considered for this review.

\section{Disease overview}

HIV-1 infection is still spreading on a worldwide level. The Joint United Nations Programme on HIV/AIDS (UNAIDS) estimates 33.2 million people are living with
HIV infection worldwide, ${ }^{2}$ the majority of whom are infected with HIV-1 and reside in subSaharan Africa and South-East Asia. Several countries of the former Soviet Union show rapidly increasing numbers of infected people, mainly in intravenous drug users. In those countries affected, HIV infection has serious consequences for both the individuals and the societies. Individually, it results in social marginalization, poverty, and inability to work and sustain the economic basis of living for those infected and their families. For societies, the loss of young adults in the midst of their productive years and the secondary costs due to healthcare and childhood poverty markedly reduce the economic development perspectives.

Despite long-standing prevention campaigns, HIV infection continues to spread in the US and Western Europe as well. Highly active antiretroviral therapy (HAART), which is widely available in developed countries, has massively improved morbidity and mortality in those infected. ${ }^{3,4}$ It can control virus replication for many years in the individual. The associated burden of healthcare costs, especially for antiretroviral drugs, is increasingly being viewed as a challenge to healthcare systems.

Table I Evidence base included in the review

\begin{tabular}{lll}
\hline Category & \multicolumn{2}{l}{ Number of records } \\
\cline { 2 - 3 } & Full papers & Abstracts \\
\hline Initial search & 114 & 57 \\
Records excluded & 93 & 46 \\
Records included & 21 & $1 \mathrm{I}$ \\
Additional studies identified & 3 & \\
Level I clinical evidence & & $\mathrm{I}$ \\
Level 2 clinical evidence & 6 & 10 \\
Level $\geq 3$ clinical evidence & $\mathrm{II}$ & \\
trials other than RCT & & \\
Case studies & $\mathrm{I}$ & \\
\hline
\end{tabular}

Notes: For definition of levels of evidence, see Core Evidence website (http://www.dovepress.com/core-evidence-journal).

Abbreviation: RCT, randomized controlled trial. 
Fortunately, the overall rate of resistance against antiretroviral drugs in treated patients appears not to increase, ${ }^{4-8}$ probably due to more rational use and a wider spectrum of agents available to achieve optimal virus inhibition. However, transmission of resistant virus strains remains a problem in newly infected individuals, ${ }^{6,9}$ resulting in suboptimal response. ${ }^{10,11}$

\section{Current therapy options}

The choice of the antiretroviral regimen is highly individualized and based on considerations such as primary resistance, the probability of treatment interruptions or nonadherence, drug interactions with HIV and concomitant medication, as well as on patient desires. There is broad consensus on the aim of achieving and maintaining a viral load that is undetectable with the most sensitive standard assays once antiretroviral therapy has been initiated. Initial treatment should comprise two nucleoside/nucleotide reverse transcriptase inhibitors. The third drug should either be an NNRTI or a protease inhibitor. There is some indication of a better virologic response with the NNRTI efavirenz, but more resistance in case of failure, often associated with cross resistance within the drug class. ${ }^{12}$ In later lines of therapy, individual tolerance and the resistance profile of the virus determine the choice of drugs. Therefore, beyond initial therapy, most drugs that retain antiviral activity, are tolerated sufficiently well, and have no unfavorable pharmacokinetic interactions are used as combination partners. In this setting, drugs are added rather than exchanged. Due to their low genetic hurdle to resistance, NNRTIs are not used frequently beyond first-or second-line therapy.

Most protease inhibitors, NNRTIs, and nucleoside/ nucleotide reverse transcriptase inhibitors are licensed for all lines of therapy. The novel, less cross-resistant protease inhibitors tipranavir and darunavir, the fusion inhibitor enfuvirtide, the R5 coreceptor blocker maraviroc, and the integrase inhibitor raltegravir, however, are currently licensed for later lines only. Accordingly, clinical trial results mainly reflect their use in salvage therapy, with an $\sim 50 \%-100 \%$ increase of virologic response rates over placebo when the respective compound is added to a background combination selected according to the patient's virus resistance genotype ("optimized background"). All NNRTIs except etravirine (ie, efavirenz, nevirapine, and delavirdine) are highly cross resistant and require only 1-2 mutations in viral reverse transcriptase in order to lose their activity almost completely (ie, they have a low "genetic hurdle"). ${ }^{13,14}$ The key NNRTI mutation $\mathrm{K} 103 \mathrm{~N}$ is rapidly selected for during failure of the most widely used NNRTI efavirenz, facilitated by reduced adherence,${ }^{15}$ and confers cross resistance to nevirapine and delavirdine. It has little impact on viral replication capacity, ${ }^{16}$ whereas protease inhibitor and nucleoside reverse transcriptor inhibitor mutations reduce it. ${ }^{17-21}$ In the absence of therapy, this loss of viral "fitness" leads to counter-selection in favor of wild-type virus and to the virtual disappearance of resistance mutations in genotypic resistance analyses. ${ }^{22}$ However, they remain present as minor variants probably indefinitely. ${ }^{23-25}$

The R5 coreceptor blocker, maraviroc, is only active in patients with no evidence of virus strains using the $\mathrm{X} 4$ coreceptor. It has demonstrated high activity in two parallel trials, in which it was added to an optimized background regimen (MOTIVATE-1 and -2). ${ }^{26}$ In later lines of therapy, however, half of the patients carry viruses using either both the $\mathrm{R} 5$ and the $\mathrm{X} 4$ coreceptor or only $\mathrm{X} 4,{ }^{27}$ rendering maraviroc inactive. Even in antiretroviral naïve patients, $18 \%$ exhibited $\mathrm{X} 4$ use. ${ }^{28}$ Moreover, the drug failed to demonstrate equivalence to efavirenz (both in combination with zidovudine and lamivudine) in antiretroviral naïve patients (MERIT study), ${ }^{29}$ probably due to emergence of X4-using variants undetected at baseline. ${ }^{30}$ Maraviroc is also a substrate of CYP3A4. Therefore, dose adjustment is necessary when combined with inducers (eg, most NNRTIs) or inhibitors of CYP3A4 (eg, ritonavir). ${ }^{31}$

Raltegravir, the first licensed HIV integrase inhibitor, has demonstrated high antiviral activity when added to an optimized background regimen in patients with virologic failure and resistant virus. ${ }^{32-34}$ In contrast to maraviroc, raltegravir showed equivalent virologic suppression when compared with efavirenz in previously untreated patients. It is mainly metabolized by UGT1A $1 ;{ }^{35}$ despite minor interactions, dose adjustment is not considered necessary in combination with other antiretroviral drugs. ${ }^{36}$

\section{Unmet needs}

Patients who develop extended antiretroviral drug resistance need as many virologically active agents as possible in order to compose a regimen with two or more active drugs. As outlined above for maraviroc, R5 inhibitors only represent an option in some patients. Moreover, in clinical routine, toxicity issues often prevent the use of some active drugs, so that the number of viable options for the individual may be substantially smaller than predicted by the resistance genotype. Therefore, there is a general need for many potentially active compounds to be available.

Development of novel compounds from within an existing class has the advantage of exploiting a well established treatment principle with less risk of unexpected toxicities than for compounds with novel modes of action. Accordingly, the 
protease inhibitors tipranavir and darunavir were developed to target viruses resistant against earlier generations of protease inhibitors.

Due to broad cross resistance within the group of NNRTIs, a promising second-line NNRTI treatment option was lacking. Such an option is attractive because it should help to overcome the limitations of the low genetic hurdle and cross resistance of efavirenz, nevirapine, and delavirdine. Furthermore, should transmitted NNRTI resistance increase, novel, less cross-resistant compounds would be needed even in early lines of therapy.

\section{Clinical evidence with etravirine}

Etravirine is a diarylpyrimidine compound that was specifically designed to bind to HIV-1 reverse trancriptase in a more flexible manner than efavirenz and nevirapine and to require more than one key NNRTI resistance mutation for resistance development ${ }^{37-39}$ (ie, to possess a higher genetic hurdle to resistance).

A phase I/II 7-day monotherapy trial in 19 HIV-infected, antiretroviral-naïve subjects showed a $-1.99 \log _{10}$ decline in HIV plasma viremia with etravirine as opposed to -0.06 $\log _{10}$ in controls. ${ }^{40}$ A similar open-label phase IIa trial in 16 individuals on a failing regimen containing efavirenz or nevirapine and with documented phenotypic NNRTI resistance yielded a median plasma HIV RNA reduction of $-0.89 \log _{10} 41$ Study TMC125-C203 was a randomized, phase IIb, placebocontrolled, two-stage trial evaluating safety, tolerability, and preliminary efficacy of three twice-daily doses of etravirine ${ }^{42}$ (400, 800, and $1200 \mathrm{mg}$ twice daily of the phase II TF035 formulation). No evidence of relevant neuropsychiatric side effects was found, and virus load suppression was superior at week 24 in the etravirine arm in stage II of the trial. ${ }^{43}$

The TMC125-C223 trial investigated two doses of etravirine (400 and $800 \mathrm{mg}$ twice daily) versus placebo, combined with an optimized background regimen in 199 patients with genotypic NNRTI resistance and at least three primary protease inhibitor resistance-associated mutations. Viral load reductions were -1.04 and $-0.18 \log _{10}$ in the etravirine arms compared with $-0.19 \log _{10}$ in the comparator arm. ${ }^{44}$

The phase IIb study, TMC125-C227, investigated the antiviral activity of etravirine in 116 treatment-experienced, protease inhibitor-naïve HIV-1 infected patients with genotypic evidence of NNRTI resistance at screening or in prior resistance analyses. The study was a randomized, exploratory, active-controlled, open-label comparison of etravirine $(n=59)$ and a protease inhibitor chosen by the investigator $(n=57)$. It was conducted in South Africa, South
America, Asia, and Europe and used a different formulation of etravirine for which, however, exposure was comparable to the phase III formulation that is currently approved..$^{45}$ The suppression rate of HIV plasma viremia to $<50$ copies $/ \mathrm{mL}$ at week 12 was superior in the control arm (52\%) compared with the etravirine arm (22\%). This is explained by a higher number of mutations conferring resistance to NNRTI and to the nucleoside analogue backbone of the regimen. ${ }^{46}$ Pretreatment definitely reduced the overall genetic hurdle to resistance against an NNRTI-based regimen in the study participants, so that fewer mutational steps had to be taken for the virus with etravirine. The hypothesis may be raised, however, that the evolutionary cost for the virus in terms of replicative fitness when acquiring new NNRTI mutations could be less than for protease inhibitor resistance mutations. This could contribute to a more rapid development of resistance on etravirine than expected. Etravirine, therefore, is unlikely to be sufficiently active in patients who have been left on a failing NNRTI-based regimen for longer and should be combined with other active compounds.

Etravirine was licensed based on the analyses of 24-week data from two randomized, double-blind, placebo-controlled, phase III trials (TMC125-C206 and TMC125-C216), generally referred to as DUET-1 and DUET-2. ${ }^{47,48}$ Both trials were designed identically and results are presented here as a pooled analysis. A total of 1203 treatment-experienced patients were randomly assigned to receive etravirine $(n=599)$ or placebo $(n=604)$ in combination with a background regimen (BR) comprising the novel protease inhibitor darunavir, pharmacologically boosted with ritonavir $100 \mathrm{mg}$ twice daily. Two additional investigator-selected agents had to be given, with or without enfuvirtide. Patients were eligible if their HIV plasma viral load was greater than 5000 copies $/ \mathrm{mL}$ while on a stable antiretroviral regimen for at least 8 weeks. In addition, patients had $\geq 1$ NNRTI resistance-associated mutations at screening or documented in a prior genotypic analysis, and $\geq 3$ of the primary protease inhibitor resistance-associated mutations D30N, V32I, L33F, M46I/L, I47A/V, G48V, I50L/V, V82A/ F/L/S/T, I84V, N88S, or L90M. Randomization was stratified according to the intended use of enfuvirtide in the BR, previous use of darunavir/ritonavir, and screening viral load. Virologic response was defined as an undetectable viral load $(<50 \mathrm{HIV}-1$ RNA copies/mL) at week 24.

In the pooled analysis, demographics and baseline characteristics, as well as de novo or previous use of enfuvirtide were balanced between the etravirine and the placebo groups.

Virologic response was significantly superior in the etravirine arm compared with placebo (59.8\% versus $40.2 \%)$. 
Plasma viremia was reduced by a mean of $-2.37 \log _{10}$ from baseline in the etravirine arm as opposed to $-1.68 \log _{10}$ in the controls. Accordingly, CD4 count increased by a mean of 81 cells $/ \mathrm{mm}^{3}$ in the etravirine arm in contrast to 64 cells $/ \mathrm{mm}^{3}$ in the placebo arm. Among patients reusing or not using enfuvirtide in the background regimen, $56.7 \%$ achieved $<50$ copies of HIV RNA per $\mathrm{mL}$ with etravirine, compared with $32.7 \%$ in the controls. The response rate was somewhat higher and the difference less pronounced when enfuvirtide was used for the first time in conjunction with etravirine (68.3\% versus $61.3 \%$ ).

In addition to these clinical trials, a recently published case report supports the view that etravirine has high antiviral activity also when combined with raltegravir, a compound that was not available in the trials described above. ${ }^{49}$

Several analyses of NNRTI resistance patterns in clinical routine samples demonstrate that currently resistance to etravirine, as predicted by interpretation algorithms, is rare. ${ }^{50-55}$

In the DUET trials, etravirine exhibited the expected toxicity pattern. Of those events occurring at a rate of $>10 \%$, only nausea (13.9\% versus $11.1 \%$ with placebo) and rash (16.9\% versus $9.3 \%$ ) were significantly more frequent with etravirine. Severe reactions such as Stevens-Johnson syndrome, hypersensitivity reactions, and erythema multiforme occurred in fewer than $0.1 \%$ of patients. The rash resembled that observed during efavirenz treatment and led to discontinuation in only $2 \%$ of all patients in phase III studies. Other, less frequent, adverse events were widely distributed and probably represent the toxicity of the background combination as well. Of note, neuropsychologic side effects that frequently limit the use of efavirenz were not reported more frequently with etravirine.

Regarding quality of life, functional status, adherence, or symptom relief, there is as yet no published information from the etravirine trials.

As a consequence of the unmet needs described above, the features of the ideal product in the setting of NNRTI failure can be described as follows.

1. Lack of cross resistance within the NNRTI drug class.

2. No pharmacokinetic interactions with other antiretroviral drugs (especially ritonavir-boosted protease inhibitors), concomitant medication (eg, anticonvulsants, lipid-lowering drugs, and antigastritic agents), and lifestyle or illicit drugs (eg, methadone, PDE-5 inhibitors).

3. Different profile of side effects (especially no neuropsychologic impairment, few allergic reactions).

4. Convenient pill count and dosing intervals ("one pill once a day"), comparable to efavirenz.

5. Lack of teratogenicity.
To what extent does etravirine fulfil these criteria?

1. It is highly active against resistant variants carrying key mutations such as K103N. However, accumulation of several NNRTI resistance mutations results in increasing degrees of cross resistance. ${ }^{56}$ The drug should therefore be considered earlier during NNRTI failure and not be withheld until patients have failed for longer. Although it is not licensed for this indication, the drug might be useful in the setting of transmitted NNRTI resistance.

2. It is mainly metabolized via CYP3A4. This results in numerous interactions, especially with protease inhibitors and the R5 coreceptor blocker, maraviroc, that have to be considered when composing a regimen. The same applies to concomitant medication that is metabolized via the same pathway. Moreover, in an aging patient population, more interaction with concomitant medication has to be expected. Some interactions are unexpected ${ }^{57}$ and preclude the use in some combinations. The lack of $\mathrm{pH}$ dependence of absorption ${ }^{58}$ and the absence of QTc prolongation, ${ }^{59}$ however, are positive features.

3. The rate of neuropsychologic side effects appears to be very low if at all different from placebo. Rash was observed with etravirine also.

4. Despite pharmacokinetic properties that would probably allow for once-daily dosing, this has not been pursued in clinical trials, mainly due to difficulties in formulation. The pill count is relatively high in comparison with the other NNRTIs, although it is comparable with ritonavirboosted protease inhibitors.

5. Etravirine does not appear to be teratogenic in animal models, ${ }^{1}$ but there are no reported observations during pregnancy.

Several aspects regarding the optimal position of etravirine in antiretroviral therapy remain to be investigated.

1. Use as a stand-alone third agent. The DUET trials have demonstrated high activity in combination with ritonavirboosted darunavir, which was a novel compound when the studies started. Many study participants had low baseline resistance to darunavir, so that the results do not only reflect the effect of etravirine. Accordingly, in the TMC125-C223 trial, etravirine was used in conjunction with a ritonavir-boosted protease inhibitor. In the TMC125-C227 trial, in which it was applied as a standalone third agent, it showed little activity. This, however, may have been a problem of patient selection. It remains to be shown if - in a setting of less extensive baseline resistance or even wild-type virus -the drug could be used as a substitute for efavirenz or nevirapine, 
eg, in subjects with transmitted NNRTI resistance or in case of intolerance.

2. Extent of pharmacokinetic interactions. Gender and ethnic differences in metabolism as well as interactions with concomitant medication have to be explored in further studies in order to provide physicians with proper dose recommendations.

3. Potential for once-daily dosing. Several antiretroviral compounds can be given once daily. For etravirine, however, the high pill burden of the current formulation represents an obstacle to this strategy.

\section{Economic evidence}

The following calculations are subject to price changes, and prices may vary in different countries. They are only meant to assess the order of magnitude of economic implications. Etravirine is currently sold at a price that results in an approximate yearly cost of \$US9500 (http://www. healthpricer.com and http://www.pharmacychecker.com, accessed for update 15.7.2009). This compares favorably with the annual cost of other drugs licensed for use in later lines of therapy: \$US1 1900 for darunavir $600 \mathrm{mg}$ qd plus \$US1, 350 for ritonavir boosting; \$US11590 for raltegravir; \$US12070 for maraviroc 150/300 mg twice daily; \$US12370 for tipranavir plus \$US2700 for ritonavir boosting; and approximately \$US29360 for enfuvirtide. It is, however, more expensive than the other compounds of the class: \$US7700 for delavirdine; \$US4360 for nevirapine; and \$US6910 for efavirenz.

Treatment with efavirenz and nevirapine was shown to be cost effective or cost saving in all lines of therapy in comparison with protease inhibitor-based regimens. ${ }^{60}$ There are, as yet, no published cost-effectiveness studies using etravirine data. Given a lower price than protease inhibitors and enfuvirtide, however, there is no indication that it could be less cost effective than enfuvirtide ${ }^{61}$ in later lines of therapy.

\section{Resource utilization}

Etravirine is currently placed in later lines of therapy for patients with NNRTI resistance and protease inhibitor pretreatment. Primary virologic failure rates are decreasing, ${ }^{62}$ as is the risk and prevalence of triple-class resistance. ${ }^{63,64}$ Triple-class failure occurs slowly ${ }^{65}$ and is lower among patients who initiated a modern HAART regimen as opposed to those initiated on suboptimal regimens. ${ }^{66}$ This currently limits the use of etravirine to a relatively small percentage of patients. Furthermore, the overall prevalence of high-level antiretroviral drug resistance is decreasing, probably due to improved therapeutic options with the novel compounds (tipranavir, darunavir, maraviroc, and raltegravir). Therefore, only a small fraction of all patients receiving antiretroviral treatment still have uncontrolled replication of NNRTI-resistant viruses at present and would be candidates for etravirine therapy. There are currently no data to support the use of etravirine as a replacement for other active compounds in a suppressive regimen, such as enfuvirtide, maraviroc, and raltegravir, which are more expensive. Replacement would be attractive for patients experiencing injection-site reactions to enfuvirtide and could help to reduce the overall cost of therapy. However, switch trials are needed in order to assess if etravirine is sufficiently active when given without a ritonavir-boosted protease inhibitor.

Assuming that these results were favorable, etravirine could also represent an option for subjects with primary, transmitted NNRTI resistance, especially since it is not always detected by standard sequencing techniques. This would affect $\sim 5 \%-10 \%$ of newly infected individuals and lead to a broader use of the compound.

\section{Patient group/population}

Antiretroviral-experienced adult patients with evidence of viral replication and HIV-1 strains resistant to a NNRTI and other antiretroviral agents are likely to benefit most from etravirine, as long as the number of NNRTI resistance mutations is limited. An analysis of a large Italian resistance database (ARCA) suggests that exposure to nevirapine is associated with a higher risk of etravirine-associated resistance mutations than efavirenz. ${ }^{55}$ Therefore, etravirine appears to be used most effectively in subjects failing on efavirenz rather than nevirapine. Although the K103N mutation alone does not confer resistance to the drug, the presence of Y181V, G190S, V179D/F/T, or three or more 2007 1AS-USA-defined NNRTI amino acid changes is associated with a markedly decreased response.

Of note, no benefit was shown in the TMC125-C227 trial, when etravirine was used only in combination with nucleoside/ nucleotide reverse transcriptase inhibitors in patients who failed an NNRTI-based regimen. Therefore, use in the absence of other active "flanking" agents should be avoided where possible.

In the absence of any unexpected severe toxicity, improved virologic suppression will likely translate into improved immune reconstitution and clinical stability.

\section{Dosage, administration, and formulation}

Etravirine (Intelence) is available as $100 \mathrm{mg}$ tablets, and is taken as two tablets twice daily following a meal. 
Ingestion in the fasted state results in a $50 \%$ reduction in systemic exposure [area under the curve (AUC)]. ${ }^{1}$ The AUC is minimally reduced by ranitidine (14\%), and increased by $41 \%$ by omeprazole, ${ }^{58}$ which appears clinically irrelevant. Etravirine is a substrate of CYP 3A4, 2C9, and 2C19. It is also an inducer of CYP3A4, but an inhibitor of $2 \mathrm{C} 9$ and $2 \mathrm{C} 19$. Therefore, a careful evaluation of drug interactions with protease inhibitors, maraviroc [dose adjustment necessary, ${ }^{67}$ and concomitant therapy is required. Etravirine should not be coadministered with tipranavir/ ritonavir [etravirine exposure reduced] ${ }^{68}$ fosamprenavir/ ritonavir [fosamprenavir exposure increased], ${ }^{69}$ and atazanavir/ritonavir [atazanavir exposure decreased], ${ }^{70}$ as well as with unboosted protease inhibitors and other NNRTIs. No dose adjustment is required for either compound in combinations with lopinavir/ritonavir. ${ }^{71}$ For darunavir/ritonavir, no impact on darunavir levels but a decrease in etravirine AUC by $37 \%$ was observed in healthy volunteers. ${ }^{72}$ Given the high antiviral activity of this combination in the DUET studies, however, no dose adjustment is recommended.

For possibly interacting concomitant medication such as antiarrhythmics and warfarin, dose adjustment and/or discontinuation should be considered. Due to its long terminal half-life of $41( \pm 20)$ hours, treatment interruption or permanent discontinuation should probably be handled in the same way as with efavirenz, ie,continuation of the other drugs for some days after discontinuation of the NNRTI. Etravirine appears to have the potential for once-daily dosing, providing that the number of pills can be reduced in a future formulation. However, as the drug is being developed for later lines of therapy in which many other components are also dosed twice daily, this matter does not appear as a limitation to its use.

Etravirine has no clinically relevant effect on the pharmacokinetics of methadone. ${ }^{73}$

\section{Place in therapy}

Etravirine has its place in therapy in patients with virus resistant to NNRTI and other antiretroviral agents carrying limited numbers of the aforementioned NNRTI resistance-associated amino acid changes. This has already been implemented in the current Department of Health and Human Services (DHHS) and European AIDS Clinical Society (EACS) guidelines for antiretroviral therapy in adults. ${ }^{74-76}$ The drug is very valuable in increasing the number of drug options available to construct a well-tolerated and fully suppressive regimen and to contribute to clinical stability or improvement.
A limitation to its use is the fact that the favorable data leading to licensure were obtained only in combinations with ritonavir-boosted protease inhibitors. Although the label allows for other combinations, these data are still lacking and should be provided by further studies. It also has to be kept in mind that, due to unfavorable pharmacokinetic interactions, the drug can not be combined freely with all ritonavir-boosted protease inhibitors (see above), which might preclude its optimal use in some patients. Nevertheless, the lack of pharmacokinetic interaction with methadone makes it uncomplicated in patients on methadone substitution.

There are currently only limited pediatric data. Exposure of children 6-17 years of age to $4 \mathrm{mg} / \mathrm{kg}$ of bodyweight twice daily resulted in exposure similar to that of adults using $200 \mathrm{mg}$ twice daily, ${ }^{77}$ but studies on antiviral efficacy are lacking. Furthermore, there is as yet no experience during pregnancy. Because of lack of reproductive toxicity in animal models at an exposure similar to therapeutic levels in humans, etravirine was labelled Pregnancy Category B ${ }^{1}$ Although its use during pregnancy cannot be encouraged, pregnancies will likely occur in women receiving etravirine. These events should be recorded and evaluated in the registry supported by the manufacturer.

It is unclear if etravirine can actually replace fully active compounds in a salvage treatment regimen rather than being added as another active component. This strategy should be investigated in clinical trials.

If the galenic preparation can be improved further and the pill burden reduced, the long half-life would probably allow for once-daily dosing. This would make the drug more attractive for first-line therapy, but it could also be valuable for later lines. This use, however, is not supported by data so far and cannot be recommended at the present time.

In conclusion, etravirine is a valuable novel tool in the armamentarium of antiretroviral drugs. It could be valuable for earlier lines of failure, without a ritonavirboosted protease inhibitor, and for primary NNRTI resistance, provided clinical trials show favorable results. Efforts should be made to further explore the potential of this drug.

\section{Disclosure}

The author has served as an investigator, speaker and advisor for Tibotec (Janssen Cilag), Merck, Sharp \& Dohme, Roche, Bristol-Myers Squibb, Pfizer, GlaxoSmithKline, Gilead, and Boehringer Ingelheim. 


\section{References}

1. Anon. INTELENCETM (etravirine) Tablets. Raritan NJ: Tibotec Therapeutics, Division of Ortho Biotech Products L.P. 2008.

2. UNAIDS (the Joint United Nations Programme on HIV/AIDS). AIDS epidemic update: December 2007. Available at: http://www.google. co.uk/search?hl=en\&q=UNAIDS.+AIDS+epidemic + update $+\% 3 \mathrm{~A}+\mathrm{D}$ ecember $+2007 \& b \operatorname{tnG}=$ Search $\&$ meta $=$

3. Palella FJ Jr, Delaney KM, Moorman AC, et al. Declining morbidity and mortality among patients with advanced human immunodeficiency virus infection. HIV Outpatient Study Investigators. $N$ Engl J Med. 1998;338:853-860.

4. Losina E, Yazdanpanah Y, Duffic-Burban S, et al. The independent effect of highly active antiretroviral therapy on severe opportunistic disease incidence and mortality in HIV-infected adults in Cote d'Ivoire. Antivir Ther. 2007:12;543-551.

5. Bezemer D, Jurriaans S, Prins M, et al. Declining trend in transmission of drug-resistant HIV-1 in Amsterdam. Aids. 2004;18:1571-1577.

6. Yerly S, von WV, Ledergerber B, et al. Transmission of HIV-1 drug resistance in Switzerland: a 10-year molecular epidemiology survey. Aids. 2007;21:2223-2229.

7. SP (SPREAD Programme). Transmission of drug-resistant HIV-1 in Europe remains limited to single classes. AIDS. 2008;22:625-630.

8. Vercauteren J, Deforche K, Theys K, et al. The incidence of multidrug and full class resistance in HIV-1 infected patients is decreasing over time (2001-2006) in Portugal. Retrovirology. 2008;5:12.

9. Masquelier B, Bhaskaran K, Pillay D, et al. Prevalence of transmitted HIV-1 drug resistance and the role of resistance algorithms: data from seroconverters in the CASCADE collaboration from 1987 to 2003. J Acquir Immune Defic Syndr. 2005;40:505-511.

10. Chaix ML, Desquilbet L, Descamps D, et al. Response to HAART in French patients with resistant HIV-1 treated at primary infection: ANRS Resistance Network. Antivir Ther. 2007;12:1305-1310.

11. Poggensee G, Kucherer C, Werning J, et al. Impact of transmission of drug-resistant HIV on the course of infection and the treatment success. Data from the German HIV-1 Seroconverter Study. HIV Med. 2007;8:511-519.

12. Riddler SA, Haubrich R, DiRienzo G, et al; for the AIDS Clinical Trials Group Study A5142 Team. Class-sparing regimens for initial treatment of HIV-1 infection. N Engl J Med. 2008;358:2095-2106.

13. Clotet B. Efavirenz: resistance and cross-resistance. Int J Clin Pract Suppl. 1999;103:21-25.

14. Delaugerre C, Rohban R, Simon A, et al. Resistance profile and cross-resistance of HIV-1 among patients failing a non-nucleoside reverse transcriptase inhibitor-containing regimen. J Med Virol. 2001;65:445-458.

15. Harrigan PR, Hogg RS, Dong WW, et al. Predictors of HIV drug-resistance mutations in a large antiretroviral-naive cohort initiating triple antiretroviral therapy. J Infect Dis. 2005;191:339-347.

16. Koval CE, Dykes C, Wang J, Demeter LM. Relative replication fitness of efavirenz-resistant mutants of HIV-1: correlation with frequency during clinical therapy and evidence of compensation for the reduced fitness of K103N + L100I by the nucleoside resistance mutation L74V. Virology. 2006;353:184-192.

17. Croteau G, Doyon L, Thibeault D, McKercher G, Pilote L, Lamarre D. Impaired fitness of human immunodeficiency virus type 1 variants with highlevel resistance to protease inhibitors. J Virol. 1997;71:1089-1096.

18. Harrigan PR, Bloor S, Larder BA. Relative replicative fitness of zidovudine-resistant human immunodeficiency virus type 1 isolates in vitro. $J$ Virol. 1998;72:3773-3778.

19. Picchio GR, Valdez H, Sabbe R, et al. Altered viral fitness of HIV-1 following failure of protease inhibitor-based therapy. J Acquir Immune Defic Syndr. 2000;25:289-295.

20. Prado JG, Franco S, Matamoros T, et al. Relative replication fitness of multi-nucleoside analogue-resistant HIV-1 strains bearing a dipeptide insertion in the fingers subdomain of the reverse transcriptase and mutations at codons 67 and 215. Virology. 2004;326:103-112.
21. Wainberg MA. The impact of the M184V substitution on drug resistance and viral fitness. Expert Rev Anti Infect Ther. 2004;2:147-151.

22. Loubser S, Balfe P, Sherman G, et al. Decay of K103N mutants in cellular DNA and plasma RNA after single-dose nevirapine to reduce mother-to-child HIV transmission. Aids. 2006; 20:995-1002.

23. Flys T, Nissley DV, Claasen CW, et al. Sensitive drug-resistance assays reveal long-term persistence of HIV-1 variants with the K103N nevirapine (NVP) resistance mutation in some women and infants after the administration of single-dose NVP: HIVNET 012. J Infect Dis. 2005;192:24-29.

24. Flys TS, Chen S, Jones DC, et al. Quantitative analysis of HIV-1 variants with the $\mathrm{K} 103 \mathrm{~N}$ resistance mutation after single-dose nevirapine in women with HIV-1 subtypes A, C, and D. J Acquir Immune Defic Syndr. 2006;42:610-613.

25. Flys TS, Donnell D, Mwatha A, et al. Persistence of K103N-containing HIV-1 variants after single-dose nevirapine for prevention of HIV-1 mother-to-child transmission. J Infect Dis. 2007; 195:711-715.

26. Hardy D, Reynes J, Konourina I, et al. Efficacy and safety of maraviroc plus optimized background therapy in treatment-experienced patients infected with CCR5-tropic HIV-1: 48-week combined analysis of the MOTIVATE studies. 15th Con Retrovir Opportun Infect, Boston. 2008. Abstract 792.

27. Wilkin TJ, Su Z, Kuritzkes DR, et al. HIV type 1 chemokine coreceptor use among antiretroviral-experienced patients screened for a clinical trial of a CCR5 inhibitor: AIDS Clinical Trial Group A5211. Clin Infect Dis. 2007;44:591-595.

28. Brumme ZL, Goodrich J, Mayer HB, et al. Molecular and clinical epidemiology of CXCR4-using HIV-1 in a large population of antiretroviral-naive individuals. J Infect Dis. 2005;192:466-474.

29. Saag M, Prudence I, Heera J, et al. A multicenter, randomized, double-blind, comparative trial of a novel CCR5 antagonist, maraviroc versus efavirenz, both in combination with Combivir (zidovudine [ZDV]/lamivudine [3TC]), for the treatment of antiretroviral naive patients infected with R5 HIV-1: Week 48 results of the MERIT study. 4th IAS Conference Sydney, Australia. 2007. Abstract WESS104.

30. Westby M, Lewis M, Whitcomb, et al. Emergence of CXCR4-using human immunodeficiency virus type 1 (HIV-1) variants in a minority of HIV-1-infected patients following treatment with the CCR5 antagonist maraviroc is from a pretreatment CXCR4-using virus reservoir. J Virol. 2006;80:4909-4920.

31. Abel S, Jenkins TM, Whitlock LA, Ridgway CE, Muirhead GJ. Effects of CYP3A4 inducers with and without CYP3A4 inhibitors on the pharmacokinetics of maraviroc in healthy volunteers. $\mathrm{Br} J$ Clin Pharmacol. 2008;65(Suppl 1):38-46.

32. Cooper DA, Gatell J, Rockstroh J, et al. Results of BENCHMRK-1, a phase III study evaluating the efficacy and safety of MK-0518, a novel HIV-1 integrase inhibitor, in patients with triple-class resistant virus. 14th Conf Retro Opportun Infect Los Angeles. 2007. Abstract $105 \mathrm{aLB}$.

33. Grinsztejn B, Nguyen BY, Katlama C, et al. Safety and efficacy of the HIV-1 integrase inhibitor raltegravir (MK-0518) in treatment-experienced patients with multidrug-resistant virus: a phase II randomised controlled trial. Lancet. 2007;369:1261-1269.

34. Steigbigel R, Kumar P, Eron J, et al. Results of BENCHMRK-2, a phase III study evaluating the efficacy and safety of MK-0518, a novel HIV-1 integrase inhibitor, in patients with triple-class resistant virus. 14th Conf Retro Opportun Infect Los Angeles, CA. 2007. Abstract $105 \mathrm{bLB}$.

35. Kassahun K, McIntosh I, Cui D, et al. Metabolism and disposition in humans of raltegravir (MK-0518), an anti-AIDS drug targeting the human immunodeficiency virus 1 integrase enzyme. Drug Metab Dispos. 2007;35:1657-1663.

36. Anon. ISENTRESS ${ }^{\mathrm{TM}}$ Tablets (package insert). Whitehouse Station NJ: Merck \& Co, 2007. 
37. Andries K, Azijn H, Thielemans T, et al. TMC125, a novel next-generation nonnucleoside reverse transcriptase inhibitor active against nonnucleoside reverse transcriptase inhibitor-resistant human immunodeficiency virus type 1. Antimicrob Agents Chemother. 2004;48:4680-4686.

38. Das K, Clark AD Jr, Lewi PJ, et al. Roles of conformational and positional adaptability in structure-based design of TMC125-R165335 (etravirine) and related non-nucleoside reverse transcriptase inhibitors that are highly potent and effective against wild-type and drug-resistant HIV-1 variants. J Med Chem. 2004;47:2550-2560.

39. Vingerhoets J, Azijn H, Fransen E, et al. TMC125 displays a high genetic barrier to the development of resistance: evidence from in vitro selection experiments. J Virol. 2005;79:12773-12782.

40. Gruzdev B, Rakhmanova A, Doubovskaya E, et al. A randomized, double-blind, placebo-controlled trial of TMC125 as 7-day monotherapy in antiretroviral naive, HIV-1 infected subjects. AIDS. 2003;17: 2487-2494.

41. Gazzard BG, Pozniak AL, Rosenbaum W, et al. An open-label assessment of TMC 125-a new, next-generation NNRTI, for 7 days in HIV-1 infected individuals with NNRTI resistance. Aids. 2003;17: F49-F54.

42. Woodfall B, Vingerhoets J, Peeters M, et al. Impact of NNRTI and NRTI resistance on the response to the regimen of TMC125 plus two NRTIs in study TMC125-C227. International Congress on Drug Therapy in HIV Infection. Glasgow, UK. 2006a. Abstract PL5.6.

43. Montaner J, Yeni P, Clumeck NN, et al. Safety, tolerability, and preliminary efficacy of 48 weeks of etravirine therapy in a phase $\mathrm{IIb}$ dose-ranging study involving treatment-experienced patients with HIV-1 infection. Clin Infect Dis. 2008;47:969-978.

44. Nadler JP, Berger DS, Blick G, et al. Efficacy and safety of etravirine (TMC125) in patients with highly resistant HIV-1: primary 24-week analysis. Aids. 2007;21:F1-F10.

45. Kakuda TN, Scholler-Gyure M, Workman C, et al. Single- and multiple-dose pharmacokinetics of etravirine administered as two different formulations in HIV-1 -infected patients. Antivir Ther. 2008a; 13:655-661.

46. Woodfall B, De Smedt G, Berckmans C, Baeten B, Peeters M. No frequent reporting of neurological or psychiatric events during TMC125 treatment. 8th International Congress on Drug Therapy in HIV Infection. Glasgow, UK. 2006b. Abstract 146.

47. Lazzarin A, Campbell T, Clotet B, et al. Efficacy and safety of TMC125 (etravirine) in treatment-experienced HIV-1-infected patients in DUET-2: 24-week results from a randomised, double-blind, placebo-controlled trial. Lancet. 2007;370:39-48.

48. Madruga JV, Cahn P, Grinsztejn B, et al. Efficacy and safety of TMC125 (etravirine) in treatment-experienced HIV-1-infected patients in DUET-1: 24-week results from a randomised, double-blind, placebocontrolled trial. Lancet. 2007;370:29-38.

49. Di Biagio A, Bruzzone B, Rosso R, et al. Successful rescue therapy with raltegravir (MK-0518) and etravirine (TMC125) in an HIV-infected patient failing all four classes of antiretroviral drugs. AIDS Patient Care STDS. 2008;22:355-357.

50. Poveda E, Garrido C, de Mendoza C, et al. Prevalence of etravirine (TMC-125) resistance mutations in HIV-infected patients with prior experience of non-nucleoside reverse transcriptase inhibitors J Antimicrob Chemother. 2007;60:1409-1410.

51. Lapadula G, Calabresi A, Castelnuovo F, et al. Prevalence and risk factors for etravirine resistance among patients failing on non-nucleoside reverse transcriptase inhibitors. Antivir Ther. 2008a;13:601-605.

52. Llibre JM, Santos JR, Puig T, et al. Prevalence of etravirine-associated mutations in clinical samples with resistance to nevirapine and efavirenz. J Antimicrob Chemother. 2008;62:909-913.

53. Lapadula G, Izzo I, Gargiulo F. Updated prevalence of genotypic resistance among HIV-1 positive patients naive to antiretroviral therapy: a single center analysis. J Med Virol. 2008b;80:747-753.

54. Molina JM, Cordoba J, Gil A, Gobernado M. Epidemiology of genotypic resistance of HIV-1 in Valencia. A 4-year study. Rev Esp Quimioter. 2007;20:346-353.
55. Di Vincenzo P, Rusconi S, Adorni F, et al. Prevalence of mutations and determinants of genotypic resistance to Etravirine (TMC125) in a large Italian resistance database (ARCA). 6th European HIV Drug Resistance Workshop, Budapest, Hungary. 2008. Abstract 59.

56. Vingerhoets J, Clotet B, Peeters M, et al. Impact of baseline NNRTI mutations on the virological response to TMC125 (etravirine; ETR) in the DUET-1 and DUET-2 Phase III clinical trials. 11th EACS European AIDS Conference, Madrid, Spain. 2007. Abstract P7.3/05.

57. Kakuda TN, Scholler-Gyure M, Woodfall BJ, et al. TMC125 in combination with other medications: summary of drug-drug interactions. 8th International Congress on Drug Therapy in HIV Infection, Glasgow, Scotland. 2006. Abstract PL5.6. 2006.

58. Schöller-Gyüre M, Kakuda TN, de Smedt G, et al. A pharmacokinetic study of etravirine (TMC125) co-administered with ranitidine and omeprazole in HIV-volunteers. Br J Clin Pharmacol. 2008a; 66:508-516

59. Peeters M, Janssen K, Kakuda TN, et al. Etravirine has no effect on QT and corrected QT interval in HIV-negative volunteers. Ann Pharmacother. 2008;42:757-765.

60. Beck EJ, Mandalia S, Youle M, et al. Treatment outcome and costeffectiveness of different highly active antiretroviral therapy regimens in the UK (1996-2002). Int J STD AIDS. 2008;19:297-304.

61. Ruof J, Dusek A, DeSpirito M, Demasi RA. Cost-efficacy comparison among three antiretroviral regimens in HIV-1 infected, treatment-experienced patients. Clin Drug Investig. 2007;27:469-479.

62. Lampe FC, Gatell JM, Staszewski S, et al. Changes over time in risk of initial virological failure of combination antiretroviral therapy: a multicohort analysis, 1996 to 2002. Arch Intern Med. 2006;166:521-528.

63. Lohse N, Obel N, Kronborg G, et al. Declining risk of triple-class antiretroviral drug failure in Danish HIV-infected individuals. Aids. 2005; $19: 815-822$

64. Di Giambenedetto S, Bracciale L, Colafigli M, et al. Declining prevalence of HIV-1 drug resistance in treatment-failing patients: a clinical cohort study. Antivir Ther. 2007;12:835-839.

65. Phillips AN, Leen C, Wilson A, et al. Risk of extensive virological failure to the three original antiretroviral drug classes over long-term follow-up from the start of therapy in patients with HIV infection: an observational cohort study. Lancet. 2007;370:1923-1928.

66. Napravnik S, Keys JR, Quinlivan EB, Wohl DA, Mikeal OV, Eron JJ Jr. Triple-class antiretroviral drug resistance: risk and predictors among HIV-1-infected patients. Aids. 2007;21:825-834.

67. Davis J, Scholler-Gyure M, Kakuda TN, et al. An open, randomized, two-period, crossover study in two cohorts to investigate the effect of steady-state TMC125 (etravirine) and the combination of TMC125/darunavir/ritonavir on the steady-state Pharmacokinetics of Oral Maraviroc in Healthy Subjects. 11th European AIDS Conference/ EACSMadrid, Spain. 2007. Abstract P4.3/02.

68. Schöller M, Kraft M, Hoetelmans R, et al. Significant decrease in TMC125 exposures when co-administered with tipranavir (boosted with ritonavir) in healthy subjects. 13th Conference on Retroviruses and Opportunistic Infections (CROI), Denver, CO. 2006. Abstract 583.

69. Schöller-Gyüre M, Woodfall B, Bollen S, Peeters M, Vandermeulen K, Hoetelmans, R. Pharmacokinetics of amprenavir and TMC125 in HIV-infected volunteers receiving TMC125with fosamprenavir/ritonavir. 46th Interscience Conference on Antimicrobial Agents and Chemotherapy. 2006a. San Francisco, CA. Abstract A-370.

70. Schöller-Gyüre M, Woodfall B, De Marez T, et al. Pharmacokinetics of TMC125 with atazanavir and atazanavir/ritonavir. 8th International Congress on Drug Therapy in HIV infection, Glasgow, UK. 2006 b. Abstract P278.

71. Piscitelli SC, Baede P, Van't Klooster G, Graham N. TMC125 does not alter lopinavir/ritonavir (LPV/RTV) pharmacokinetics in healthy volunteers. 42nd Intersci Conf Antimicrob Agents Chemother San Diego, CA. 2002. Abstract A-1824.

72. Schöller-Gyüre M, Kakuda TN, Sekar V, et al. Pharmacokinetics of darunavir/ritonavir and TMC125 alone and coadministered in HIVnegative volunteers. Antivir Ther. 2007;12:789-796. 
73. Schöller-Gyüre M, van den BW, Kakuda TN, et al. Pharmacokinetic and pharmacodynamic study of the concomitant administration of methadone and TMC125 in HIV-negative volunteers. J Clin Pharmacol. 2008b;48:322-329.

74. PE (Panel of Experts). Guidelines for the Clinical Management and Treatment of HIV Infected Adults in Europe. European AIDS Clinical Society (EACS). 2007. Available at: http://www.eacs.eu/guide/index.htm.

75. Clumeck N, Pozniak A, Raffi F. European AIDS Clinical Society (EACS) guidelines for the clinical management and treatment of HIV-infected adults. HIV Med. 2008;9:65-71.
76. PAGAA (Panel on Antiretroviral Guidelines for Adults and Adolescents). Guidelines for the use of antiretroviral agents in HIV-1-infected adults and adolescents. Department of Health and Human Services. 2008. Available at: http://www.aidsinfo. nih. gov/Guidelines/GuidelineDetail.aspx? MenuItem=Guide lines\&GuidelineID=7.

77. Kakuda T, Konigs C, Feiterna-Sperling C, et al. Pharmacokinetics of the next-generation NNRTI TMC125 in HIV-infected children between 6 and 17 years of age. 15th Conference on Retroviruses and Opportunistic Infections (CROI), Boston, MA. 2008b. Abstract 578.

\section{Publish your work in this journal}

Core Evidence is an international, peer-reviewed open-access journal evaluating the evidence underlying the potential place in therapy of drugs throughout their development lifecycle from preclinical to postlaunch. The focus of each review is to evaluate the case for a new drug or class in outcome terms in specific indications and patient

\section{Dovepress}

groups. The manuscript management system is completely online and includes a very quick and fair peer-review system, which is all easy to use. Visit http://www.dovepress.com/testimonials.php to read real quotes from published authors. 